\title{
Manifestações músculo-esqueléticas como apresentação inicial das leucemias agudas na infância
}

\author{
Musculoskeletal manifestations as the onset of acute leukemias in childhood \\ Cássia Maria Passarelli Lupoli Barbosa ${ }^{1}$, Cláudia Nakamura ${ }^{2}$, Maria Teresa Terreri ${ }^{3}$, \\ Maria Lúcia de Martino Lee ${ }^{4}$, Antonio Sergio Petrilli ${ }^{5}$, Maria Odete Esteves Hilário ${ }^{6}$
}

\section{Resumo}

Objetivo: estudar a prevalência e as características clínicas e laboratoriais dos pacientes com manifestações músculo-esqueléticas na apresentação inicial das leucemias agudas.

Métodos: estudo de casos prevalentes, retrospectivo, descritivo, no qual foram avaliados pacientes com diagnóstico de leucemia aguda, atendidos no Instituto de Oncologia Pediátrica da UNIFESP, de novembro de 1999 a fevereiro de 2000. As queixas músculoesqueléticas foram investigadas através de um questionário. Os dados referentes ao exame físico e provas laboratoriais, no início da doença, foram obtidos através da revisão de prontuários.

Resultados: sessenta e uma crianças foram incluídas neste estudo, sendo que $93 \%$ apresentavam leucemia linfóide aguda, e $7 \%$ leucemia mielóide aguda. Trinta e oito crianças (62\%) apresentaram dor músculo-esquelética no início da doença. Artrite foi observada em 8 casos (13\%). A média de articulações acometidas foi 2,5 (variando de 1 a 6), sendo as mais acometidas os joelhos, os tornozelos e os cotovelos. Três pacientes $(4,9 \%)$ apresentavam hemograma normal, 54 (88\%) hemoglobina baixa (em 6 pacientes foi a única alteração), leucopenia em 14 (22\%), leucocitose em 26 (42\%), e plaquetopenia em $46(75 \%)$ pacientes. Oito pacientes (13\%) mostravam blastos em sangue periférico.

Conclusão: as queixas músculo-esqueléticas são manifestações iniciais freqüentes das leucemias agudas, e devem ser consideradas no diagnóstico diferencial da dor em membros e das artrites agudas ou crônicas da infância. Os exames laboratoriais podem ser inicialmente normais, tornando ainda mais difícil esta diferenciação.

J Pediatr (Rio J) 2002;78(6):481-4: manifestações músculoesqueléticas, artrite, dor em membros, leucemias, crianças.

\begin{abstract}
Objective: to study the frequency, the clinical features and laboratory exams of patients with musculoskeletal symptoms at the onset of acute leukemia.

Methods: retrospective, descriptive study including patients diagnosed with acute leukemia, and treated at the Institute of Pediatric Oncology of UNIFESP, carried out from November 1999 to February 2000. The data on musculoskeletal complaints were obtained from a questionnaire. The medical records were revised in order to get data on the clinical examination and laboratory tests at the beginning of the illness.
\end{abstract}

Results: sixty-one children were included in this trial, 93\% with acute lymphoid leukemia, and $7 \%$ with acute myeloid leukemia. Thirty-eight patients $(62 \%)$ had musculoskeletal pain at the onset. Arthritis was observed in 8 patients. The mean number of involved joints was $2.5(1-6)$. The most frequently involved joints were the knees, ankles and elbows. Three patients $(4.9 \%)$ had normal blood count. Low hemoglobin was reported in 54 patients $(88 \%$ ) (in 6 patients it was the only hematological abnormality), leukopenia in $14(22 \%)$, leukocytosis in $26(42 \%)$ and low platelet count in 46 (75\%).

Conclusions: the musculoskeletal symptoms are common at the onset of acute leukemia so, malignancy should always be ruled out in patients presenting chronic or acute arthritis or benign limb pain. The laboratory tests may be normal at the onset of the illness, making differential diagnosis more difficult.

J Pediatr (Rio J) 2002;78(6):481-4: musculoskeletal abnormalities, arthritis, limp pain, leukemia, children.

1. Pós-graduanda do setor de Reumatologia Pediátrica, Disciplina de Alergia, Imunologia Clínica e Reumatologia do Dep. de Pediatria da UNIFESP-EPM.

2. Especializanda da Disciplina de Alergia, Imunologia Clínica e Reumatologia do Departamento de Pediatria da UNIFESP-EPM.

3. Prof ${ }^{a}$ Assistente do Setor de Reumatologia Pediátrica da Disciplina de Alergia, Imunologia Clínica e Reumatologia do Dep. de Pediatria da UNIFESP-EPM.

4. Mestre em Pediatria, Hematologista Pediátrica do Instituto de Oncologia Pediátrica - GRAACC - UNIFESP-EPM.

5. Prof. Adjunto do Departamento de Pediatria. Diretor Geral do Instituto de Oncologia Pediátrica - GRAACC - UNIFESP-EPM.

6. Prof ${ }^{a}$ Associada e chefe da Disciplina de Alergia, Imunologia Clínica e Reumatologia do Departamento de Pediatria da UNIFESP- EPM. 


\section{Introdução}

A dor articular e a dor em membros são queixas comuns na população pediátrica, afetando 10 a $20 \%$ das crianças em idade escolar ${ }^{1}$, mas, muitas vezes, o diagnóstico diferencial pode tornar-se difícil. Sabemos que a maioria dos casos de dor em membros é de origem benigna ${ }^{2}$, porém causas orgânicas devem ser investigadas. Uma possibilidade preocupante, que deve ser sempre considerada, é a de doença neoplásica, em especial as leucemias. Recentemente, Trapani e cols. ${ }^{3}$ estudaram a incidência de câncer oculto em 1.254 crianças que se apresentavam com sintomas músculo-esqueléticos, acompanhadas por um período de 10 anos, e encontraram menos que $1 \%$ de neoplasias, sendo a mais freqüente a leucemia linfóide aguda.

A leucemia linfóide aguda (LLA) é o câncer mais comum na infância, correspondendo a $25 \%$ das neoplasias de 0 a 14 anos, e constitui $85 \%$ das leucemias infantis ${ }^{4}$. Seu pico de incidência é de 2 a 5 anos de idade, discretamente mais freqüente no sexo masculino e em indivíduos da raça branca ${ }^{4}$. O quadro clínico é bastante heterogêneo, variando de acordo com a intensidade do comprometimento medular e extramedular. Fadiga, letargia e perda de peso fazem parte dos sintomas, e 53\% apresentam febre ${ }^{5}$. Cerca de $40 \%$ dos pacientes apresentam dor óssea (difusa ou localizada) inicialmente intermitente, tendendo a tornar-se persistente, e/ou artralgia e/ou artrite, cujo padrão é bastante variável ${ }^{5}$. Segundo a literatura, artrite ocorre em uma freqüência de $13,5 \%{ }^{6}$, sendo mais comum na LLA. Pacientes com esta manifestação freqüentemente têm hemogramas iniciais normais ${ }^{5,7,8}$, o que pode contribuir para o atraso do diagnóstico. Ao exame físico, é possível não se encontrar nada além de artrite. Palidez e presença de sangramento mucocutâneo podem ser observados. O fígado, o baço e os linfonodos estão aumentados em mais da metade dos pacientes.

Com a evolução da doença, podem ocorrer, ainda, acometimento ocular, testicular, nódulos subcutâneos, aumento das glândulas salivares, priapismo e síndromes compressivas medulares.

As leucemias apresentam, portanto, sintomas e sinais inespecíficos que podem simular o quadro clínico de muitas patologias, entre elas a artrite reumatóide juvenil(ARJ ${ }^{7,9}$, febre reumática $(\mathrm{FR})^{10}$, lupus eritematoso sistêmico $(\mathrm{LES})^{11}$, púrpura trombocitopênica idiopática (PTI), aplasia medular e mononucleose infecciosa, entre outras.

Alguns trabalhos têm descrito as manifestações reumatológicas das leucemias, uma vez que queixas músculoesqueléticas podem ser sua manifestação inicial ${ }^{7,12-16}$. A artrite em leucemias pode ser crônica ou recorrente, e geralmente apresenta-se de forma pauciarticular assimétrica, geralmente aditiva, mas pode ser também migratória e seu início pode ser súbito ou insidioso ${ }^{6}$.

Os exames laboratoriais mostram diferentes alterações, refletindo o grau de infiltração medular. Anemia, usualmente normocrômica e normocítica, com baixa contagem de reticulócitos, ocorre em mais de $75 \%$ dos casos ${ }^{4}$. A contagem leucocitária pode variar de 100 a 1 milhão de leucócitos $/ \mathrm{mm}^{3}, 53 \%$ apresentam menos que $10.000,30 \%$, de 10 a 49.000, e $17 \%$, mais que 50.000 leucócitos $/ \mathrm{mm}^{3} 17$. Embora a plaquetopenia seja um achado freqüente, $46 \%$ dos pacientes com leucemia e manifestação músculo-esquelética inicial apresentam contagens normais ${ }^{8}$. A identificação de blastos no sangue periférico pode ser feita em 1/3 dos pacientes ${ }^{8}$.

Devido aos achados clínicos inespecíficos e ao retardo no aparecimento de alterações hematológicas, pode haver um atraso no diagnóstico da leucemia, em períodos descritos na literatura de 2 semanas a 13 meses ${ }^{7,13,15}$. Desta forma, hemogramas seriados são indicados para detectar alterações precoces nos casos com suspeita clínica de leucemia.

Uma vez que o diagnóstico precoce é fator determinante no tratamento e evolução das leucemias, nosso objetivo é alertar pediatras e reumatologistas para esta possibilidade em pacientes com queixas articulares e/ou dor em membros, verificando quais são os fatores que, associados, possam nos direcionar para este diagnóstico.

\section{Objetivo}

Estudar a prevalência e as características clínicas e laboratoriais dos pacientes com manifestações músculoesqueléticas, na apresentação inicial das leucemias agudas.

\section{Casuística e métodos}

Foram avaliados 61 pacientes com diagnóstico de leucemia, atendidos no Instituto de Oncologia Pediátrica (IOP) - UNIFESP, no período de novembro de 1999 a fevereiro de 2000.

Após consentimento informado, os pacientes e seus pais foram entrevistados por reumatologista pediátrico, para preenchimento do questionário, nos dias de suas consultas no IOP, ocasião em que se fez a revisão de seus prontuários, confirmando os dados de história e exame físico. Os exames laboratoriais considerados foram os primeiros a serem realizados no início da doença.

\section{Resultados}

Dos 61 pacientes com diagnóstico de leucemia, 54\% eram do sexo masculino, e $55,7 \%$, da raça caucasóide. A idade de início dos sintomas variou de 10 meses a 15 anos e 6 meses, com média de 8,1 anos. O tipo de leucemia mais freqüente foi a LLA $(93,4 \%)$. O tempo de início dos sintomas até o diagnóstico variou de 5 dias a 7 meses, com média de 2 meses. Dos 61 pacientes, 7 estavam em acompanhamento com outro diagnóstico, 1 como dor em membros benigna, 1 como LES, 2 ARJ, 1 PTI e 2 como Calazar, 3 deles em uso de corticoesteróides. 
A Tabela 1 mostra os sinais e sintomas músculo-esqueléticos na apresentação da leucemia. Trinta e oito pacientes (62\%) apresentaram dor em membros e/ou articular. A artrite detectada pelo exame físico ocorreu em cerca de $13 \%$ dos pacientes, sendo predominantemente pauciarticu$\operatorname{lar}(75 \%)$. O número de articulações acometidas variou de 1 a 6 , com média de 2,5, e as principais articulações acometidas foram joelhos, tornozelos e cotovelos. O período da dor foi predominantemente diurno ( $50 \%$ dos casos). Alterações cutâneas (púrpura) e hepatoesplenomegalia ocorreram em $28(46 \%)$ e $7(28 \%)$ pacientes, respectivamente.

Tabela 1 - Sinais e sintomas músculo-esqueléticos na apresentação da leucemia $(n=61)$

\begin{tabular}{lcc}
\hline Sinais e sintomas & $\mathbf{N}$ & $\mathbf{\%}$ \\
\hline Dor em membros e/ou articular & 38 & 62 \\
$\quad$ diurna & 19 & 50 \\
$\quad$ noturna & 4 & 10,5 \\
$\quad$ contínua & 15 & 39,4 \\
Dificuldade para deambular & 24 & 39,3 \\
Artrite (anamnese) & 14 & 22,9 \\
Artrite ao exame físico & 8 & 13,1 \\
\hline
\end{tabular}

A Tabela 2 mostra os exames laboratoriais. Devemos ressaltar que 3 pacientes apresentavam hemograma completamente normal, e 6 , apenas anemia $(<11 \mathrm{~g} \%)$, variando de 7,1 a 10,9 g\%. A velocidade de hemossedimentação (VHS) foi maior que $50 \mathrm{~mm}$ em todos os pacientes pesquisados $(7 / 61)$.

Tabela 2 - Exames laboratoriais realizados na apresentação da leucemia $(n=61)$

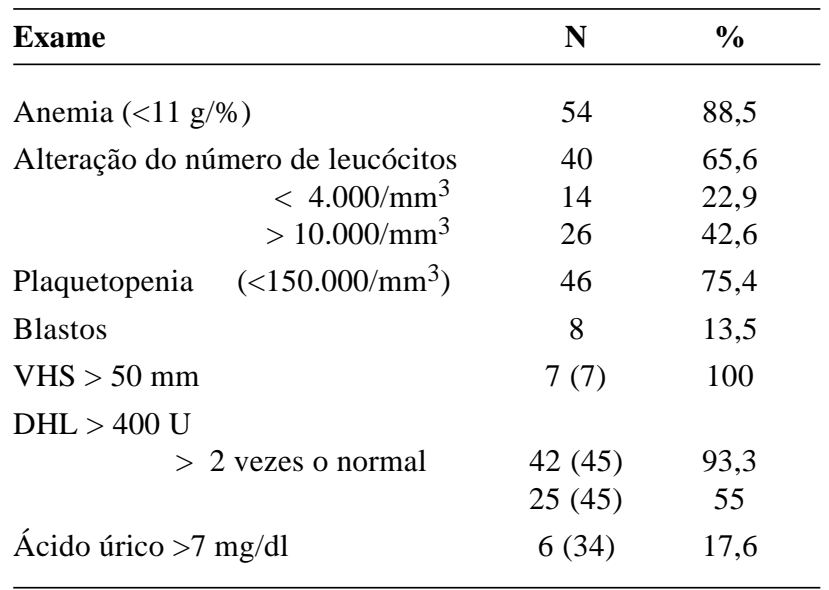

VHS: velocidade de hemossedimentação, DHL: desidrogenase láctica
A dosagem de desidrogenase láctica (DHL) foi realizada em 45 pacientes, encontrando-se valores alterados em 42 $(93,3 \%)$, sendo que alcançou valores bastante altos (até $6.000 \mathrm{UI}$ ), e em $55 \%$ dos pacientes esteve 2 vezes acima dos valores normais.

\section{Discussão}

Tem sido muito bem documentado que sintomas músculo-esqueléticos podem ser a manifestação inicial das leucemias em crianças.

O tempo decorrido desde o início da queixa reumática até o diagnóstico de leucemia variou de 5 dias a 7 meses, com média de 2 meses. As doenças reumatológicas, em especial a febre reumática e a artrite reumatóide juvenil, são importantes diagnósticos diferenciais das leucemias, e cinco dos 61 pacientes descritos foram diagnosticados erroneamente, retardando a introdução da terapêutica adequada.

A incidência de sintomas, ou achados reumatológicos, como apresentação das neoplasias, é muito variável, e a sua importância como "marcadores" de câncer ainda não foi suficientemente avaliada ${ }^{12,13,18}$. Há vários trabalhos na literatura que observaram a freqüência e características deste tipo de manifestação inicial nas doenças linfoproliferativas $7,8,15,16$. No nosso estudo, ocorreram em $62 \%$ dos casos, semelhante ao apresentado na literatura estudada. Sabe-se que as leucemias com apresentação clínica mais insidiosa apresentam estas queixas com maior freqüência.

A dor foi mais frequientemente diurna, o que difere da literatura, e a dor característica que acorda a criança durante a noite foi observada em $10,5 \%$ dos $\operatorname{casos}^{7,12,14}$. A dor articular severa, desproporcional aos sinais flogísticos, como ocorre na febre reumática, também é uma característica das leucemias, levando o paciente ao leito, ou à cadeira de rodas. No nosso estudo, 39,3\% dos pacientes apresentaram dificuldade para deambular. Tuten e cols. ${ }^{18}$ estudaram 77 crianças com leucemia e observaram que $11,6 \%$ tinham como queixa inicial a claudicação.

Como descrito na literatura, a artrite foi predominantemente de membros inferiores e pauciarticular $(75 \%)^{6}$. Nas leucemias agudas, as articulações dos joelhos, como ocorreu em nosso estudo, são as mais acometidas, porém articulações de tornozelos, punhos, cotovelos e quadris também são descritas ${ }^{7,12}$.

O hemograma pode ser normal nas fases iniciais ${ }^{3,7,8}$, como ocorreu em 3 de 61 pacientes, ou apresentar apenas anemia de leve a moderada, sem alterações na série branca ou plaquetária.

Há necessidade de que o hemograma seja repetido, para detectar alterações precoces sugestivas de leucemia. Leucocitose ou leucopenia, como ocorreu, respectivamente, 
em cerca de $42 \%$ e $23 \%$ de nossos pacientes, nos alertam para leucemias, principalmente se acompanhadas de linfocitose. O número de pacientes com blastos na periferia, no início da doença, de $13 \%$, foi inferior ao descrito na literatura, de $33 \%{ }^{7}$, isto talvez se deva ao fato dos hemogramas iniciais terem sido feitos rotineiramente em laboratórios, e não por hematologista, com a intenção de encontrar estas células.

A plaquetopenia é outro dado relevante para o diagnóstico das leucoses, uma vez que a ARJ sistêmica, que é um diagnóstico diferencial importante entre as doenças reumatológicas, cursa com plaquetas normais, ou com trombocitose. Plaquetas abaixo de $150.000 / \mathrm{mm}^{3}$ foram encontradas em $75 \%$ dos casos, o que é superior à literatura ${ }^{8}$.

A velocidade de hemossedimentação se mostrou alterada em $100 \%$ dos casos e, por ser exame inespecífico, não é de auxílio no diagnóstico diferencial.

Dos 45 pacientes de nossa casuística, nos quais se dosou DHL, 93,3\% apresentaram níveis maiores que os normais (>400), sendo que $55 \%$ com valores superiores a 2 vezes o normal. Wallendal et al. ${ }^{19}$ estudaram 12 crianças que tiveram diagnóstico de neoplasia maligna e artralgia, ou artrite, na apresentação da doença e compararam com 24 pacientes com diagnóstico de ARJ. Os autores encontraram que, no grupo das neoplasias, os níveis de DHL foram de 2,2 vezes o normal, e em crianças com ARJ, apenas 0,8 vezes. Portanto, crianças com sintomas consistentes de ARJ e que apresentem níveis elevados de DHL devem ser submetidas à investigação para neoplasia.

O uso de corticoesteróides em crianças com artrite e suspeita de colagenose, mesmo sem diagnóstico definitivo, pode retardar a definição da leucemia, porque alivia os sintomas e pode alterar a citologia e histologia da medula óssea.

\section{Conclusão}

As queixas músculo-esqueléticas são manifestações iniciais freqüentes das leucemias agudas na infância e devem ser consideradas no diagnóstico diferencial da dor em membros e das artrites agudas e crônicas.

Os exames laboratoriais iniciais podem ser absolutamente normais, ou mostrar alterações discretas que não direcionam para o diagnóstico de neoplasia. É necessário que se tenha em mente este diferencial, para que possamos acompanhar de perto estas crianças e solicitar exames seriados, até que o diagnóstico esteja claramente definido. A dosagem de DHL pode ser útil para nos ajudar no diagnóstico diferencial.

\section{Referências bibliográficas}

1. Goodman JE, McGrath PJ. The epidemiology of pain in children and adolescents: a review. Pain 1991;46:247-64.

2. Bowyer SL, Hollister RJ. Dores em membros em crianças. In: Levine MD. Clínicas Pediátricas da América do Norte 1984;5: 1105-35.

3. Trapani S, Grisolia F, Simonini G, Calabri GB, Falcini F. Incidence of occult cancer in children presenting with musculoskeletal symptoms: A 10-year survey in a pediatric rheumatology unit. Seminars in arthritis and rheumatism 2000; 29(6):348-59.

4. Lee MLM. Leucemia linfóide aguda. In: Carvalho ES, Carvalho WB. Terapêutica e Prática Pediátrica 2000;1251-5.

5. Pui CH, Crist WM. Acute lymphoblastic leukemia. In: Pui CH. Childhood Leukemias. $1^{a}$ ed. Cambridge: Cambridge University Press; 1999.p.288-312.

6. Spilberg I, Meyer GJ. The arthritis of leukemia. Arthritis Rheum 1972;15(6):630-5.

7. Ostrov BE, Goldsmith DP, Athreya BH. Differentiation of systemic juvenile rheumatoid arthritis from acute leukemia near the onset of disease. J Pediar 1993;122:595-8.

8. Jonsson OG, Sartain P, Ducore JM, Buchanan GR. Bone pain as an initial symptom of childhood acute lymphoblastic leukemia: association with nearly normal hematologic indexes. J Pediatr 1990;117:233-7.

9. Sills EM. Errors in diagnosis of juvenile rheumatoid arthritis. Hopkins Med. J 1973;133:88-5.

10. Oliveira SKF, Laun L. Artrite como primeira manifestação de leucemias em crianças. J Pediatr 1989;65(1/2):36-1.

11. Salsbury FT, Sabio H, Conrad D, Kesler RW, Levien MG. Acute leukemia with features of systemic lupus erythematosus. J Pediatr 1984;105(1):57-9.

12. Schaller J. Arthritis as a presenting manifestation of malignancy in children. J Pediatr 1972;81(4):793-7.

13. Cabral DA, Tucker L. Malignancies in children who initially present with rheumatic complaints. J Pediatr 1999;134:53-57.

14. Costello PB, Brecher ML, Starr JI, Freeman AI, Green FA. A prospective analysis of the frequency, course, and possible prognostic significance of the joint manifestations of childhood leukemia. J Rheumatol 1983;10:753-7.

15. Bradlow A, Barton C. Arthritic presentation of childhood leukemia. Postgrad Med J 1991;67:562-4.

16. Fink CW, Windmiller J, Sartain P. Arthritis as the presenting feature of childhood leukemia. Arthritis Rheum 1972;15:347-9.

17. Margolin JF, Poplack DG. Acute lymphoblastic leukemia. In: Pizzo PA, Poplach DG. Principles and Practice of Pediatric Oncology. Philadelphia: Lippincott Williams \& Wilkins Publishers; 2002.p. 14 .

18. Tuten RH, Gabos PG, Kumar J, Harter GD. The limping child: a manifestation of acute leukemia. J Pediatr Orthop 1998; 18:625-9.

19. Wallendal M, Stork L, Hollister JR. The discriminating value of serum lactate dehydrogenase levels in children with malignant neoplasms presenting as joint pain. Arch Pediatr Adolesc Med 1996;150:70-3.

Endereço para correspondência:

Dra. Cássia Maria P.L. Barbosa

Rua Itagyba Santiago, 360 - apto 63 - Vila Alexandria

CEP 04635-051 - São Paulo, SP

Fone: (11) 5666.0801 - Fax: (11) 5666.1195

E-mail: cassia@vicnet.com.br 